\title{
A general approach to the sign problem - the factorization method with multiple observables
}

\author{
Konstantinos N. Anagnostopoulos ${ }^{1}$ * Takehiro Azuma ${ }^{2}$ 团 and Jun Nishimura ${ }^{3,4}$ \\ ${ }^{1}$ Physics Department, National Technical University of Athens, Zografou Campus, GR-15780 Athens, Greece \\ ${ }^{2}$ Institute for Fundamental Sciences, Setsunan University, \\ 17-8 Ikeda Nakamachi, Neyagawa, Osaka, 572-8508, Japan \\ ${ }^{3}$ KEK Theory Center, High Energy Accelerator Research Organization, Tsukuba 305-0801, Japan \\ ${ }^{4}$ Department of Particle and Nuclear Physics, School of High Energy Accelerator Science, \\ Graduate University for Advanced Studies (SOKENDAI), Tsukuba 305-0801, Japan
}

(Dated: February, 2011; preprint: KEK-TH-1399)

\begin{abstract}
The sign problem is a notorious problem, which occurs in Monte Carlo simulations of a system with the partition function whose integrand is not real positive. The basic idea of the factorization method applied on such a system is to control some observables in order to determine and sample efficiently the region of configuration space which gives important contribution to the partition function. We argue that it is crucial to choose appropriately the set of the observables to be controlled in order for the method to work successfully in a general system. This is demonstrated by an explicit example, in which it turns out to be necessary to control more than one observable. Extrapolation to large system size is possible due to the nice scaling properties of the factorized functions, and known results obtained by an analytic method are shown to be consistently reproduced.
\end{abstract}

PACS numbers: 05.10.Ln, 02.70.Tt, 11.15.Ha

\section{INTRODUCTION}

Monte Carlo simulation is a powerful tool for studying statistical systems from first principles. When the partition function has an integrand which is not real positive, however, one encounters a notorious technical problem called the sign problem. There have been many proposals, but most of them are successful only for a very special system [1] or for a very small region of the parameter space [2]. In Ref. [3], two of the authors proposed a method termed the factorization method, which is based on the factorization property of the density of states of properly chosen physical observables. It has been tested in a random matrix theory for finite density QCD [4], and applied also to the lattice QCD at finite density [5, 6] with some important new ideas [6]. In the lattice gauge theory, a lot of efforts are actively pursued also using other methods such as analytic continuation [7], multiparameter reweighting [8], complex Langevin dynamics [9] and the Taylor expansion method [10].

The basic idea of the factorization method is to control some observables in order to determine and sample efficiently the region of configuration space which gives important contribution to the partition function. While the previous studies [3 -6] suggest its potential usefulness in a wider range of applications, the choice of the observables to be controlled seemed rather arbitrary. In this work we argue that it is actually crucial to choose them appropriately in order for the method to work suc-

\footnotetext{
*Electronic address: konstant@mail.ntua.gr

${ }^{\dagger}$ Electronic address: azuma@mpg.setsunan.ac.jp

${ }^{\ddagger}$ Electronic address: jnishi@post.kek.jp
}

cessfully in a general system. With this new insight, we consider that the factorization method has become a very promising approach applicable to any interesting system that suffers from the sign problem.

\section{SIGN PROBLEM}

Let us consider a general system defined by the partition function

$$
Z=\int d A \mathrm{e}^{-S_{0}[A]+i \Gamma[A]},
$$

where $A$ represents the dynamical variables, and $S_{0}$ and $\Gamma$ represent the real part and the imaginary part of the action, respectively. Since the integrand of (1D) is not real positive due to $\Gamma$, one cannot view it as a sampling probability in a Monte Carlo simulation. One way to calculate the expectation value of an observable $\mathcal{O}$ is to use the reweighting formula

$$
\langle\mathcal{O}\rangle=\frac{\left\langle\mathcal{O} \mathrm{e}^{i \Gamma}\right\rangle_{0}}{\left\langle\mathrm{e}^{i \Gamma}\right\rangle_{0}},
$$

where the expectation values on the right-hand side are taken with respect to the phase-quenched model

$$
Z_{0}=\int d A \mathrm{e}^{-S_{0}[A]}
$$

which can be simulated in the usual manner. The expectation value $\left\langle\mathrm{e}^{i \Gamma}\right\rangle_{0}$ is nothing but the ratio of the partition functions $Z / Z_{0}$. Therefore it decreases exponentially for large system size $V$ as $\mathrm{e}^{-V \Delta f}$, where $\Delta f>0$ represents the difference in the free energy density of the two systems. This can happen due to huge cancellations from 
$\mathrm{e}^{i \Gamma}$, and occurs also in the numerator of (2). As a result one needs $\mathrm{O}\left(\mathrm{e}^{\text {const. } V}\right)$ configurations to compute the expectation value $\langle\mathcal{O}\rangle$ with given accuracy. This is called the sign problem.

There is also a general problem called the "overlap problem" in using a reweighting formula like (2). This occurs since the region $R_{0}$ of configuration space one can sample effectively by simulating the phase-quenched model has very little overlap with the region $R$ which gives important contribution in the evaluation of $\left\langle\mathcal{O} e^{i \Gamma}\right\rangle_{0}$ and $\left\langle\mathrm{e}^{i \Gamma}\right\rangle_{0}$. One has to run a very long simulation until one can sample enough configurations in the region $R$.

\section{FACTORIZATION METHOD}

In order to reduce the overlap problem, we control some observables so that one can sample configurations in the region $R$ efficiently. Let us introduce a set of such observables

$$
\Sigma=\left\{\mathcal{O}_{k} \mid k=1, \cdots, n\right\}
$$

and define the normalized observables

$$
\widetilde{\mathcal{O}}_{k}=\frac{\mathcal{O}_{k}}{\left\langle\mathcal{O}_{k}\right\rangle_{0}}
$$

Their expectation values can be written as

$$
\left\langle\tilde{\mathcal{O}}_{j}\right\rangle=\int\left[\prod_{k=1}^{n} d x_{k}\right] x_{j} \rho\left(x_{1}, \cdots, x_{n}\right)
$$

using the density of states

$$
\rho\left(x_{1}, \cdots, x_{n}\right)=\left\langle\prod_{k=1}^{n} \delta\left(x_{k}-\widetilde{\mathcal{O}}_{k}\right)\right\rangle .
$$

Applying the reweighting formula to (7), one can easily derive the factorization property

$$
\rho\left(x_{1}, \cdots, x_{n}\right)=\frac{1}{C} \rho^{(0)}\left(x_{1}, \cdots, x_{n}\right) w\left(x_{1}, \cdots, x_{n}\right),
$$

where $C=\left\langle\mathrm{e}^{i \Gamma}\right\rangle_{0}$ and

$$
\rho^{(0)}\left(x_{1}, \cdots, x_{n}\right)=\left\langle\prod_{k=1}^{n} \delta\left(x_{k}-\widetilde{\mathcal{O}}_{k}\right)\right\rangle_{0}
$$

is the density of states for the phase-quenched model. The correction factor $w\left(x_{1}, \cdots, x_{n}\right)$ is given by

$$
w\left(x_{1}, \cdots, x_{n}\right)=\left\langle\mathrm{e}^{i \Gamma}\right\rangle_{x_{1}, \cdots, x_{n}}
$$

as an expectation value in a constrained system

$$
Z\left(x_{1}, \cdots, x_{n}\right)=\int d A \mathrm{e}^{-S_{0}} \prod_{k=1}^{n} \delta\left(x_{k}-\widetilde{\mathcal{O}}_{k}\right)
$$

In what follows we assume that $w\left(x_{1}, \cdots, x_{n}\right)$ is real and positive [1].

When the system size $V$ goes to $\infty$, the expectation values are given by $\left\langle\widetilde{\mathcal{O}}_{k}\right\rangle=\bar{x}_{k}$, where $\left(\bar{x}_{1}, \cdots, \bar{x}_{n}\right)$ denotes the position of the peak of $\rho\left(x_{1}, \cdots, x_{n}\right)$. Hence they can be obtained by solving

$$
f_{k}^{(0)}\left(x_{1}, \cdots, x_{n}\right)=-\frac{\partial}{\partial x_{k}} \Phi\left(x_{1}, \cdots, x_{n}\right)
$$

for $k=1, \cdots, n$, where we have defined

$$
\begin{aligned}
& f_{k}^{(0)}\left(x_{1}, \cdots, x_{n}\right)=\lim _{V \rightarrow \infty} \frac{1}{V} \frac{\partial}{\partial x_{k}} \log \rho^{(0)}\left(x_{1}, \cdots, x_{n}\right) \\
& \Phi\left(x_{1}, \cdots, x_{n}\right)=\lim _{V \rightarrow \infty} \frac{1}{V} \log w\left(x_{1}, \cdots, x_{n}\right) .
\end{aligned}
$$

Note that the right-hand side of (12), which represents the effect of $\Gamma$, can largely shift the peak from that of $\rho^{(0)}\left(x_{1}, \cdots, x_{n}\right)$, which is given by $x_{k}=1$ due to the chosen normalization (5). Thus we can obtain $\left\langle\widetilde{\mathcal{O}}_{k}\right\rangle$ including the contribution from configurations that are difficult to sample by simulating the phase-quenched model without any constraints.

\section{CHOICE OF OBSERVABLES}

What is written above is mathematically correct for an arbitrary choice of the set $\Sigma$ of observables. We will argue, however, that the overlap problem can still occur in the evaluation of (10) by simulating (11). Note that the only difference from evaluating the denominator of (2) is the existence of the constraints. In fact it turns out to be important to choose the set $\Sigma$ appropriately so that the overlap problem is removed.

In order to clarify the overlap problem in the evaluation of (10), let us consider another observable $\mathcal{O}_{n+1}$, and define the corresponding functions $\rho^{(0)}$ and $w$ with $n$ replaced by $n+1$. Then we obtain the relation

$$
\begin{aligned}
& w\left(x_{1}, \cdots, x_{n}\right) \\
& =\frac{\int d x_{n+1} \rho^{(0)}\left(x_{1}, \cdots, x_{n+1}\right) w\left(x_{1}, \cdots, x_{n+1}\right)}{\int d x_{n+1} \rho^{(0)}\left(x_{1}, \cdots, x_{n+1}\right)} .
\end{aligned}
$$

When one simulates (11), one mostly samples configurations with $\widetilde{\mathcal{O}}_{n+1}$ close to $\left\langle\widetilde{\mathcal{O}}_{n+1}\right\rangle_{x_{1}, \cdots, x_{n}}$. However, it can happen that the integration over $x_{n+1}$ in the numerator of (14) has important contribution from the region of $x_{n+1}$ not close to $\left\langle\widetilde{\mathcal{O}}_{n+1}\right\rangle_{x_{1}, \cdots, x_{n}}$ if $w\left(x_{1}, \cdots, x_{n+1}\right)$ has strong dependence on $x_{n+1}$. In that case, one clearly has some remaining overlap problem in obtaining $w\left(x_{1}, \cdots, x_{n}\right)$ by simulating (11). The overlap problem can be reduced further by including the observable $\mathcal{O}_{n+1}$ in the set $\Sigma$. One can, in principle, repeat this procedure until the overlap problem is totally removed.

Let us then discuss what is the minimal set of observables that is sufficient to remove the overlap problem. 
For that, let $\Sigma$ be a set of all possible observables, and assume that there is no more overlap problem. Here we consider a simplified situation, in which the right-hand side of (12) is nonzero for $1 \leq k \leq K$, and vanishes for the rest; i.e.,

$$
\frac{\partial}{\partial x_{j}} \Phi\left(x_{1}, \cdots, x_{n}\right)=0 \quad \text { for } K<j \leq n
$$

near the peak $\left(\bar{x}_{1}, \cdots, \bar{x}_{n}\right)$. Then we can actually use a smaller set $\Sigma^{\prime}=\left\{\mathcal{O}_{k} \mid k=1, \cdots, K\right\}$ without having the overlap problem in evaluating $w\left(x_{1}, \cdots, x_{K}\right)$ around the peak. Furthermore, from (15) one can easily show that the expectation value $\left\langle\widetilde{\mathcal{O}}_{j}\right\rangle$ for $j>K$ can be evaluated as $\left\langle\widetilde{\mathcal{O}}_{j}\right\rangle \simeq\left\langle\widetilde{\mathcal{O}}_{j}\right\rangle_{\bar{x}_{1}, \cdots, \bar{x}_{K}}$. That this happens in spite of the sign problem can be understood by noting that

$$
\left\langle\widetilde{\mathcal{O}}_{j} \mathrm{e}^{i \Gamma}\right\rangle_{\bar{x}_{1}, \cdots, \bar{x}_{K}} \simeq\left\langle\widetilde{\mathcal{O}}_{j}\right\rangle_{\bar{x}_{1}, \cdots, \bar{x}_{K}}\left\langle\mathrm{e}^{i \Gamma}\right\rangle_{\bar{x}_{1}, \cdots, \bar{x}_{K}} ;
$$

namely the observables $\widetilde{\mathcal{O}}_{j}(j>K)$ are decorrelated with $\mathrm{e}^{i \Gamma}$. The fluctuation of the phase plays an important role in the determination of $\left(\bar{x}_{1}, \cdots, \bar{x}_{K}\right)$ through the saddlepoint equation (12), but once they are determined, the phase can be neglected completely when evaluating the expectation values of the observables $\widetilde{\mathcal{O}}_{j}(j>K)$.

In general, we can expect (15) to hold only approximately. In that case the systematic error involved in the above evaluation of $\left\langle\widetilde{\mathcal{O}}_{j}\right\rangle$ is given by $(j, l>K)$

$$
\begin{aligned}
\Delta \bar{x}_{j} & =\left.\left(\mathcal{H}^{-1}\right)_{j l} \frac{\partial}{\partial x_{l}} \Phi\left(x_{1}, \cdots, x_{n}\right)\right|_{\bar{x}_{1}, \cdots, \bar{x}_{n}}, \\
\mathcal{H}_{j l} & \left.\equiv \frac{\partial}{\partial x_{l}} f_{j}^{(0)}\left(x_{1}, \cdots, x_{n}\right)\right|_{\bar{x}_{1}, \cdots, \bar{x}_{n}} .
\end{aligned}
$$

This may also cause a small overlap problem in evaluating $w\left(x_{1}, \cdots, x_{K}\right)$ around the peak.

A practical way to search for the observables to be included in the set $\Sigma$ is to calculate an ensemble average of $\mathrm{e}^{i \Gamma}$ in the phase-quenched model with an observable $\widetilde{\mathcal{O}}$ fixed to $x$. If it becomes much larger in some region of $x$, the observable should be considered as a candidate. We expect that there are many systems in which only a few observables have to be included in the set $\Sigma$.

\section{AN EXPLICIT EXAMPLE}

Let us demonstrate how the method works in an explicit example. Here we study a matrix model defined by the partition function [12]

$$
\begin{aligned}
Z & =\int d A \mathrm{e}^{-S_{\mathrm{b}}}(\operatorname{det} \mathcal{D})^{N_{\mathrm{f}}}, \\
S_{\mathrm{b}} & =\frac{1}{2} N \sum_{\mu=1}^{4} \operatorname{tr}\left(A_{\mu}\right)^{2}, \quad \mathcal{D}=\sum_{\mu=1}^{4} \Gamma_{\mu} \otimes A_{\mu},
\end{aligned}
$$

where $A_{\mu}(\mu=1, \cdots, 4)$ are $N \times N$ Hermitian matrices and the $2 \times 2$ matrices $\Gamma_{\mu}$ are Pauli matrices $\Gamma_{j}=\sigma_{j}$ for $j=1,2,3$, and $\Gamma_{4}=i \mathbf{1}$. The system has a rotational $\mathrm{SO}(4)$ symmetry corresponding to $A_{\mu} \mapsto O_{\mu \nu} A_{\nu}$ with $O \in \mathrm{SO}(4)$. The determinant $\operatorname{det} \mathcal{D}$ is complex in general. Under parity transformation $A_{4} \rightarrow-A_{4}, A_{j} \rightarrow A_{j}(j \neq$ 4 ), it transforms as $\operatorname{det} \mathcal{D} \rightarrow(\operatorname{det} \mathcal{D})^{*}$. This implies that $\operatorname{det} \mathcal{D}$ is real for configurations with $A_{4}=0$. From this fact alone, it follows that the phase of the determinant becomes stationary for configurations with $A_{4}=A_{3}=0$ since one cannot have a phase fluctuation within a linear perturbation around such configurations 13 .

This model was proposed [12] as a toy model for the spontaneous symmetry breaking (SSB) of the $\mathrm{SO}(10)$ rotational symmetry expected to occur in the IKKT model, a conjectured nonperturbative formulation of superstring theory 14. In the IKKT model, the spacetime is represented by the eigenvalue distribution of $A_{\mu}$ $(\mu=1, \cdots, 10)$ [15, 16], and the $\mathrm{SSB}$ of $\mathrm{SO}(10)$ realizes a scenario for dynamical compactification of extra dimensions in superstring theory. This scenario is supported by explicit calculations based on the Gaussian expansion method (GEM) 19 21]. It is considered [13] that the SSB is induced by the phase of the complex Pfaffian obtained by integrating out the fermionic variables.

In the model (19), the $\mathrm{SO}(4)$ rotational symmetry is expected to be spontaneously broken in the large- $N$ limit with $r=N_{\mathrm{f}} / N$ fixed, where $N_{\mathrm{f}}$ is the exponent in (19). As an order parameter, we consider the "moment of inertia tensor"

$$
T_{\mu \nu}=\frac{1}{N} \operatorname{tr}\left(A_{\mu} A_{\nu}\right),
$$

and its real positive eigenvalues $\lambda_{k}(k=1, \cdots, 4)$ ordered as $\lambda_{1} \geq \lambda_{2} \geq \lambda_{3} \geq \lambda_{4}$. If their expectation values turn out to be unequal in the large- $N$ limit, it implies the SSB of the $\mathrm{SO}(4)$ symmetry.

The model (19) was studied by the GEM up to the ninth order for $r \leq 2$ 222. It was found that the $\mathrm{SO}(4)$ symmetry is spontaneously broken down to $\mathrm{SO}(2)$. Furthermore, by controlling the eigenvalues to have a hierarchy $\lambda_{d} \gg \lambda_{d+1}$, one can obtain $d$-dimensional configurations $(d=1,2,3)$, for which the phase fluctuations become milder according the arguments below Eq. (20). These properties of the model makes it an ideal testing ground for the ideas in Secs. III and IV.

At $r=1$, for instance, the results obtained by GEM are $\left\langle\lambda_{1}\right\rangle=\left\langle\lambda_{2}\right\rangle \simeq 2.1,\left\langle\lambda_{3}\right\rangle \simeq 1.0,\left\langle\lambda_{4}\right\rangle \simeq 0.8$, whereas $\left\langle\lambda_{k}\right\rangle_{0}=\frac{3}{2}$ for the phase-quenched model. Therefore, the expectation values of observables normalized as (5) are

$$
\left\langle\tilde{\lambda}_{1}\right\rangle=\left\langle\tilde{\lambda}_{2}\right\rangle \simeq 1.4, \quad\left\langle\tilde{\lambda}_{3}\right\rangle \simeq 0.7, \quad\left\langle\tilde{\lambda}_{4}\right\rangle \simeq 0.5 .
$$

\section{MONTE CARLO SIMULATION}

We study the model (19) at $r=1$, where the sign problem is severe. Since we know that the eigenvalues $\lambda_{k}$ have strong correlation with the fluctuation of $\Gamma$, we use $\mathcal{O}_{k}=\lambda_{k}(k=1, \cdots, 4)$ as the observables in the set 
$\Sigma$. Searching for the solution to (12) in its full generality is a formidable task. Here we simply check that the GEM result (22) is indeed a solution to the saddle-point equation (12). This is sufficient for demonstrating that the method is error free and that the generalization of the factorization method proposed in this work is necessary in order to remove the remaining overlap problem. The question whether one can successfully determine the absolute maximum of $\rho\left(x_{1}, \ldots, x_{4}\right)$ is subject to a more detailed investigation.

Let us therefore assume that the $\mathrm{SO}(2)$ symmetry remains, which implies $x_{1}=x_{2}$. Equation (12) then reduces to

$$
\frac{\partial}{\partial x_{k}} \log \rho_{\mathrm{SO}(2)}^{(0)}\left(x_{2}, x_{3}, x_{4}\right)=-\frac{\partial}{\partial x_{k}} \log w_{\mathrm{SO}(2)}\left(x_{2}, x_{3}, x_{4}\right)
$$

for $k=2,3,4$, where we have defined

$$
\begin{aligned}
& \rho_{\mathrm{SO}(2)}^{(0)}(x, y, z)=\rho^{(0)}(x, x, y, z) \\
& w_{\mathrm{SO}(2)}(x, y, z)=w(x, x, y, z) .
\end{aligned}
$$

In order to be brief [23], let us only discuss Eq. (23) for $k=2$. We set $x_{3}=0.7$ and $x_{4}=0.5$, and solve the equation for $x_{2}$. We also define $w(x)=w_{\mathrm{SO}(2)}(x, 0.7,0.5)$ and $\rho^{(0)}(x)=\rho_{\mathrm{SO}(2)}^{(0)}(x, 0.7,0.5)$.

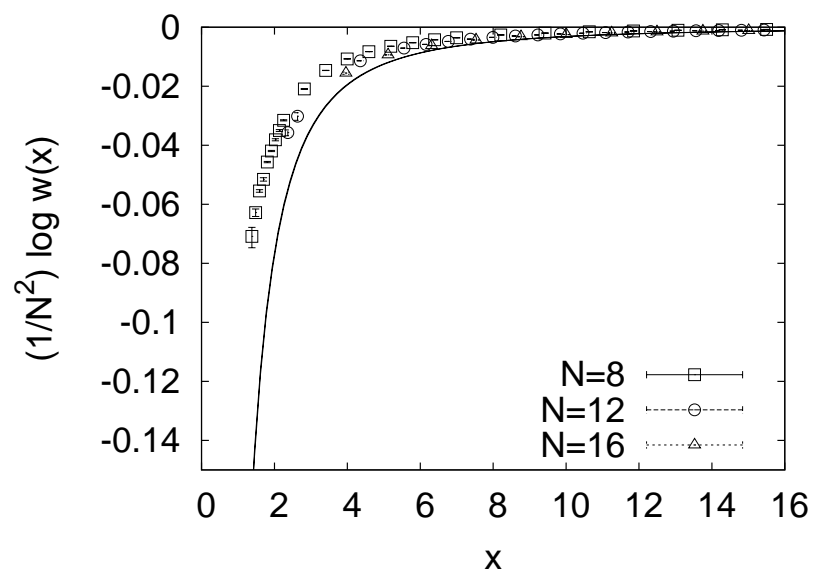

FIG. 1: The function $\frac{1}{N^{2}} \log w(x)$ is plotted for $N=8,12,16$. The solid line represents the asymptotic behavior (25) with the coefficients extrapolated to $N=\infty$.

In Fig. 11we plot our Monte Carlo data for $\frac{1}{N^{2}} \log w(x)$. It approaches zero at large $x$, where the dominant configurations have $\lambda_{2} \gg \lambda_{3}$, and the phase $\Gamma$ becomes stationary due to the argument below Eq. (20). One can actually show that the asymptotic behavior of $w(x)$ at large $x$ is

$$
\frac{1}{N^{2}} \log w(x) \simeq-c_{1} x^{-2}+c_{2} x^{-5 / 2}
$$

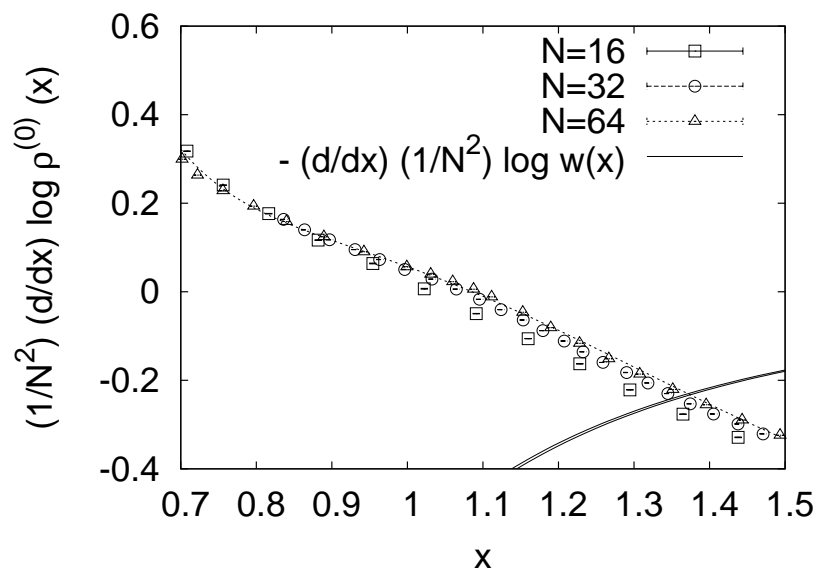

FIG. 2: The function $\frac{1}{N^{2}} \frac{d}{d x} \log \rho^{(0)}(x)$ is plotted for $N=$ $16,32,64$. The dashed line is drawn to guide the eye. We also plot $-\frac{d}{d x} \lim _{N \rightarrow \infty}\left\{\frac{1}{N^{2}} \log w(x)\right\}$ obtained from Fig. 11 where the two solid lines show the margin of error. The value of $x$ at the intersecting point is consistent with the GEM result $\left\langle\tilde{\lambda}_{2}\right\rangle \simeq 1.4$.

Fitting our data to (25) for $N=8,12,16$, we find that finite $N$ effects in the coefficients $c_{1}$ and $c_{2}$ are consistent with $\mathrm{O}(1 / N)$. Making extrapolations to $N=\infty$ based on this observation, we obtain $c_{1}=0.322(2)$ and $c_{2}=$ 0.021(1). The solid line in Fig. 1 represents Eq. (25) with these extrapolated values. Figure 2 shows that the solution is $x=1.373(2)$, which is consistent with the GEM result $\left\langle\tilde{\lambda}_{2}\right\rangle \simeq 1.4$.

Let us then consider what happens if we add

$$
\mathcal{O}=-\frac{1}{N} \sum_{\mu \neq \nu} \operatorname{tr}\left[A_{\mu}, A_{\nu}\right]^{2}
$$

as the fifth observable in the set $\Sigma$. We define the corresponding functions $\rho^{(0)}$ and $w$ with five arguments, and also define the reduced functions

$$
\begin{aligned}
\rho_{\mathcal{O}}^{(0)}(x) & =\rho^{(0)}(1.4,1.4,0.7,0.5, x), \\
w_{\mathcal{O}}(x) & =w(1.4,1.4,0.7,0.5, x),
\end{aligned}
$$

which correspond to fixing $\tilde{\lambda}_{k}$ to the GEM result (22), and $\widetilde{\mathcal{O}}=\mathcal{O} /\langle\mathcal{O}\rangle_{0}$ to some value $x$. We find that $\frac{1}{N^{2}} \log w_{\mathcal{O}}(x)$ approaches zero as $x \rightarrow 0$ [24] with the asymptotic behavior

$$
\frac{1}{N^{2}} \log w_{\mathcal{O}}(x)=-d_{1} x^{2}+d_{2} x^{5 / 2} .
$$

Therefore, the observable $\mathcal{O}$ may, in principle, be a "dangerous" one which must be included in the set $\Sigma$. Figure 3 is a plot obtained similarly to Fig. 2, which shows that the effect of the phase represented by (17) is to shift the estimate of $\langle\widetilde{\mathcal{O}}\rangle$ by $\Delta \bar{x}=0.07(3)$. On the other hand, the standard deviation of the distribution $\rho_{\mathcal{O}}^{(0)}(x)$ is estimated as $\sigma \sim 0.7 / N$ from the slope of the function 
plotted in Fig. 3 around $x \sim 0.92$. This means that the deviation $\Delta \bar{x}$ is $\lesssim 2 \sigma$ for $N \leq 16$. Thus, the remaining overlap problem in obtaining the data in Fig. 1 is practically small as far as this observable (26) is concerned. The fact that we are able to reproduce the GEM result with only four observables $\lambda_{k}$ in the set $\Sigma$ suggests that the remaining overlap problem is indeed not so severe.

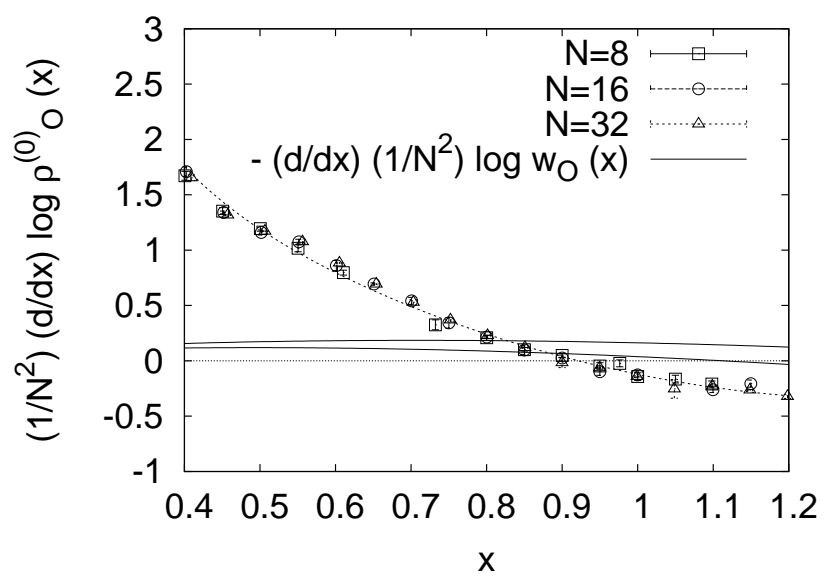

FIG. 3: The function $\frac{1}{N^{2}} \frac{d}{d x} \log \rho_{\mathcal{O}}^{(0)}(x)$ is plotted for $N=$ $8,16,32$. The dashed line is drawn to guide the eye. We also plot $-\frac{d}{d x} \lim _{N \rightarrow \infty}\left\{\frac{1}{N^{2}} \log w_{\mathcal{O}}(x)\right\}$, where the two solid lines show the margin of error. From this figure, we find that the position of the peak shifts from $x=0.92$ to $x=0.85(3)$ due to the effect of the phase.

\section{SUMMARY AND DISCUSSION}

In this work we have discussed a general approach to systems with the sign problem based on the factorization property (8) of the density of states. The method aims at reducing the overlap problem by controlling the observables which have strong correlation with the phase $\mathrm{e}^{i \Gamma}$. Once the solution to the saddle-point equation (12) is obtained for such observables, all the other observables can be investigated without the sign problem. We have presented an explicit example, in which Monte Carlo data suggests that one has to control only a few observables to remove the overlap problem almost completely. We speculate that this is the case in many interesting systems. Finding the minimal set of observables for each system as described in Section IV would be the subject of a future investigation.

The main task of the method is to calculate the function (10) by simulating (11), which still suffers from cancellations due to $\mathrm{e}^{i \Gamma}$. However, near the solution of the saddle-point equation (12), the fluctuation of $\Gamma$ becomes milder than in the phase-quenched system without constraints. Hence it is expected in many cases that one can compute the function (10), directly or by using asymptotic behaviors such as (25), for reasonable system size. Then the scaling properties represented by (13) enable extrapolations to infinite system size. We hope that Monte Carlo studies of many interesting systems that are hindered by the sign problem can be made possible by using the factorization method.

\section{ACKNOWLEDGMENTS}

We would like to thank T. Aoyama, M. Hanada, N. Kawashima and M. Oshikawa for useful discussions and comments. Computations have been carried out on PC clusters at KEK and NTUA. The work of K.N.A. was partially funded by a PEVE2010 grant from NTUA. The work of J.N. is supported in part by Grant-in-Aid for Scientific Research (No. 19340066 and 20540286) from Japan Society for the Promotion of Science.
[1] W. Bietenholz, A. Pochinsky and U. J. Wiese, Phys. Rev. Lett. 75, 4524 (1995).

[2] For a recent review, see M. P. Lombardo, K. Splittorff and J. J. M. Verbaarschot, arXiv:0912.4410, P. de Forcrand, PoS LAT2009, 010 (2009).

[3] K. N. Anagnostopoulos and J. Nishimura, Phys. Rev. D 66, 106008 (2002).

[4] J. Ambjorn, K. N. Anagnostopoulos, J. Nishimura and J. J. M. Verbaarschot, JHEP 10, 062 (2002); Phys. Rev. D 70, 035010 (2004).

[5] Z. Fodor, S. D. Katz and C. Schmidt, JHEP 03, 121 (2007).

[6] S. Ejiri, Phys. Rev. D 77, 014508 (2008); arXiv:1009.1186.

[7] P. de Forcrand and O. Philipsen, Nucl. Phys. B 642, 290 (2002); M. D'Elia and M. P. Lombardo, Phys. Rev. D 67, 014505 (2003).

[8] Z. Fodor and S. D. Katz, Phys. Lett. B 534, 87 (2002);
JHEP 04, 050 (2004).

[9] G. Aarts and I. O. Stamatescu, JHEP 09, 018 (2008); G. Aarts and F. A. James, JHEP 08, 020 (2010).

[10] C. R. Allton et al., Phys. Rev. D 66, 074507 (2002); R. V. Gavai and S. Gupta, Phys. Rev. D 68, 034506 (2003).

[11] This is the case in the models studied in Refs. [3] and [5]. See Ref. [4] for an analysis in the case where $w$ can flip its sign.

[12] J. Nishimura, Phys. Rev. D 65, 105012 (2002).

[13] J. Nishimura and G. Vernizzi, JHEP 04, 015 (2000); Phys. Rev. Lett. 85, 4664 (2000).

[14] N. Ishibashi, H. Kawai, Y. Kitazawa and A. Tsuchiya, Nucl. Phys. B 498, 467 (1997); for a comprehensive review see e.g. H. Aoki, S. Iso, H. Kawai, Y. Kitazawa, A. Tsuchiya and T. Tada, Prog. Theor. Phys. Suppl. 134, 47 (1999); T. Azuma, hep-th/0401120.

[15] H. Aoki, S. Iso, H. Kawai, Y. Kitazawa and T. Tada, 
Prog. Theor. Phys. 99, 713 (1998).

[16] Numerical simulations of matrix models of the IKKT type have provided a wealth of information on the large$N$ limit and the nonperturbative dynamics of their degrees of freedom that are, in particular, related to the emergent space-time geometry [3, 17]. Such simulations have been extended recently to shed light on important problems such as demonstrating the gauge/gravity duality from first principles and understanding the microscopic description of black hole thermodynamics in terms of string degrees of freedom [18].

[17] J. Ambjorn, K. N. Anagnostopoulos, W. Bietenholz, T. Hotta and J. Nishimura, JHEP 07, 013 (2000); JHEP 07, 011 (2000); J. Ambjorn, K. N. Anagnostopoulos, W. Bietenholz, F. Hofheinz and J. Nishimura, Phys. Rev. D 65, 086001 (2002); P. Bialas, Z. Burda, B. Petersson and J. Tabaczek, Nucl. Phys. B 592, 391 (2001); Z. Burda, B. Petersson and J. Tabaczek, Nucl. Phys. B 602, 399 (2001); Z. Burda, B. Petersson and M. Wattenberg, JHEP 03, 058 (2005).

[18] M. Hanada, J. Nishimura, S. Takeuchi, Phys. Rev. Lett. 99, 161602 (2007); S. Catterall and T. Wiseman, JHEP 12, 104 (2007); K. N. Anagnostopoulos, M. Hanada, J. Nishimura and S. Takeuchi, Phys. Rev. Lett. 100, 021601 (2008); S. Catterall and T. Wiseman, Phys. Rev. D 78, 041502 (2008); M. Hanada, Y. Hyaku- take, J. Nishimura and S. Takeuchi, Phys. Rev. Lett. 102, 191602 (2009); M. Hanada, A. Miwa, J. Nishimura and S. Takeuchi, Phys. Rev. Lett. 102, 181602 (2009); M. Hanada, J. Nishimura, Y. Sekino and T. Yoneya, Phys. Rev. Lett. 104, 151601 (2010); S. Catterall and T. Wiseman, JHEP 04, 077 (2010); for a review see e.g. J. Nishimura, PoS LAT2009, 016 (2009).

[19] J. Nishimura and F. Sugino, JHEP 05, 001 (2002).

[20] H. Kawai, S. Kawamoto, T. Kuroki, T. Matsuo and S. Shinohara, Nucl. Phys. B 647, 153 (2002); H. Kawai, S. Kawamoto, T. Kuroki and S. Shinohara, Prog. Theor. Phys. 109, 115 (2003); T. Aoyama and H. Kawai, Prog. Theor. Phys. 116, 405 (2006).

[21] T. Aoyama, J. Nishimura and T. Okubo, Prog. Theor. Phys. 125, 537 (2011).

[22] J. Nishimura, T. Okubo and F. Sugino, Prog. Theor. Phys. 114, 487 (2005).

[23] K.N. Anagnostopoulos, T. Azuma, J. Nishimura, work in progress. Details of our calculations for all $k$ and for the $\mathrm{SO}(3)$ ansatz will be presented there.

[24] As $x$ decreases, the dominant configurations have the property $\left[A_{\mu}, A_{\nu}\right] \approx 0$, meaning that $A_{\mu}$ are simultaneously diagonalizable, e.g. as $A_{\mu}=\operatorname{diag}\left(\alpha_{\mu}^{(1)}, \cdots, \alpha_{\mu}^{(N)}\right)$. For such configurations, the determinant becomes $\operatorname{det} \mathcal{D}=\prod_{i=1}^{N}\left\{\sum_{\mu}\left(\alpha_{\mu}^{(i)}\right)^{2}\right\} \geq 0$. 